\title{
Dissecting the Molecular Interactions between Wheat and the Fungal Pathogen Zymoseptoria tritici
}

\author{
Graeme J. Kettles and Kostya Kanyuka* \\ Department of Plant Biology and Crop Science, Rothamsted Research, Harpenden, UK
}

The Dothideomycete fungus Zymoseptoria tritici (previously known as Mycosphaerella graminicola and Septoria tritici) is the causative agent of Septoria tritici leaf blotch (STB) disease of wheat (Triticum aestivum L.). In Europe, STB is the most economically damaging disease of wheat, with an estimated $\sim € 1$ billion per year in fungicide expenditure directed toward its control. Here, an overview of our current understanding of the molecular events that occur during $Z$. tritici infection of wheat leaves is presented. On the host side, this includes the contribution of (1) the pathogen-associated molecular pattern-triggered immunity (PTI) layer of plant defense, and (2) major Stb loci for resistance against $Z$. tritici. On the pathogen side of the interaction, we consolidate evidence from recent bioinformatic, transcriptomic and proteomic studies that begin

OPEN ACCESS

Edited by:

Mark Findlay Belmonte,

University of Manitoba, Canada

Reviewed by:

William Underwood,

United States Department of Agriculture-Agricultural Research

Service, USA

Biswapriya Biswavas Misra

University of Florida, USA

*Correspondence:

Kostya Kanyuka kostya.kanyuka@rothamsted.ac.uk

Specialty section:

This article was submitted to Plant Biotic Interactions,

a section of the journal

Frontiers in Plant Science

Received: 01 February 2016 Accepted: 30 March 2016 Published: 15 April 2016

Citation:

Kettles GJ and Kanyuka K (2016) Dissecting the Molecular Interactions between Wheat and the Fungal Pathogen Zymoseptoria tritici. Front. Plant Sci. 7:508 doi: 10.3389/fpls.2016.00508 to explain the contribution of $Z$. tritici effector proteins to the biphasic lifestyle of the fungus. This includes the discovery of chitin-binding proteins in the $Z$. tritici secretome, which contribute to evasion of immune surveillance by this pathogen, and the possible existence of 'necrotrophic' effectors from $Z$. tritici, which may actively stimulate host recognition in a manner similar to related necrotrophic fungal pathogens. We finish by speculating on how some of these recent fundamental discoveries might be harnessed to help improve resistance to STB in the world's second largest food crop.

Keywords: Septoria tritici blotch (STB), Mycosphaerella graminicola, effector, pathogen-associated molecular pattern (PAMP), chitin, disease resistance, PAMP-triggered immunity (PTI), wheat

\section{THE Zymoseptoria tritici LIFE CYCLE AND PATHOGENESIS PROGRAM}

Zymoseptoria tritici is an ascomycete fungus belonging to the family Mycosphaerellaceae in the class Dothideomycetes. The $Z$. tritici lifestyle is described as hemibiotrophic, with two distinct phases of infection. Following inoculation onto the leaf surface by rain splash, spores germinate and the fungus invades exclusively through the stomata before undergoing a prolonged asymptomatic phase of very slow hyphal growth in the apoplastic space between mesophyll cells. There is no evidence of host cell penetration or formation of specialized feeding structures such as haustoria (Kema et al., 1996). This phase has also often been refered to as 'biotrophic.' Recent transcriptomic and metabolic profiling of $Z$. tritici infection of susceptible wheat, however, indicated that the fungus' own lipids and fatty acids are most likely used as the main energy sources during this phase. That said, a number of fungal genes encoding secreted cutinases and lipases were found to be significantly up-regulated during the asymptomatic phase, and it has been proposed that host lipids (e.g., leaf surface waxes) may also be utilized (Rudd et al., 2015). This first infection phase is also characterized by the absence, 
or very weak defense response (Keon et al., 2007; Yang et al., 2013b; Rudd et al., 2015) suggesting that Z. tritici is able to successfully suppress or avoid immune elicitation. However, the ratio of fungal to wheat biomass at this stage is extremely low and therefore any local host defense response (if present) may be diluted out and be undetectable by current methods. The asymptomatic phase typically lasts 7-10 days following inoculation depending on the particular cultivarisolate combination, after which there is a rapid transition to the symptomatic phase, which is frequently referred to as 'necrotrophic.' This second infection phase is typified by the large-scale reprogramming of both host and pathogen transcriptomes, a strong activation of host defense responses culminating in apoptotic-like rather than necrotic cell death and release of nutrients into the leaf apoplast and, as a consequence, a substantial build-up of fungal biomass (Keon et al., 2007; Yang et al., 2013b; Rudd et al., 2015). Macroscopically visible dead leaf areas then expand to form irregularly-shaped blotches (lesions) in which fungal asexual sporulation structures called pycnidia develop. These erupt through stomatal openings and release pycnidiospores that may initiate further rounds of infection when spread to healthy tissues via rain splash.

\section{Z. tritici EFFECTORS AND HOST CELL SURFACE IMMUNE RECEPTORS PLAY KEY ROLES DURING THE ASYMPTOMATIC INFECTION PHASE}

The principle function of the immune system is to recognize non-self molecules that betray the presence of an invader. Non-self molecules that trigger innate immune responses are considered pathogen-associated molecular patterns (PAMPs) (Jones and Dangl, 2006). Several fungal PAMPs have been described, including chitin (polymer of long chains of $N$-acetylglucosamine), $\beta$-glucans (polymers of glucose), mannans (polymers derived from mannose, galactose, and glucose) derived from cell walls, and ergosterol found in cell membranes (Klarzynski et al., 2000; Lochman and Mikes, 2006; McGreal et al., 2006; Shetty et al., 2009; Sánchez-Vallet et al., 2014). Successful immune activation following PAMP elicitation leads to PAMP-triggered immunity (PTI) (Jones and Dangl, 2006).

For a successful pathogen to usurp host immunity, the invader must deploy secreted effector proteins, metabolites or other mechanisms to overcome host defenses triggered during PTI. Of particular importance to fungal-plant interactions (including the Z. tritici - wheat interaction) is the PAMP chitin, which represents one of the major components of fungal cell walls and is naturally absent in plants (Sánchez-Vallet et al., 2014). Chitin functions as an elicitor of defense in both dicots and monocots, including a number of model and crop species, such as rice and wheat. Preventing or suppressing chitin-triggered defense is therefore likely to be important for any fungal pathogen. The Ecp6 effector from the fungus Cladosporium fulvum that causes the tomato leaf mold disease (Bolton et al.,
2008) was demonstrated to have ultrahigh chitin-binding affinity via cooperation of two out of its three lysin motifs (LysM) (de Jonge et al., 2010). Z. tritici encodes three LysM-containing proteins (Mg1LysM, Mg3LysM, and MgxLysM) with Mg3LysM being the functional ortholog of C. fulvum Ecp6. It has been recently demonstrated that both Mg1LysM and Mg3LysM are transcriptionally highly up-regulated during symptomless colonization of wheat and both bind chitin (Marshall et al., 2011). However, only Mg3LysM is capable of blocking chitininduced elicitation of wheat defenses (Figure 1). Curiously, both Mg3LysM and Mg1LysM also have a unique protective effect for Z. tritici, shielding the fungal cell wall from digestion by host chitinases (Marshall et al., 2011). This activity has not been observed for LysM effectors from other fungi including Ecp6. These results demonstrate the importance of chitin-binding effectors during the early stage of plant colonization.

Molecular recognition of chitin fragments in cereals requires the Chitin Elicitor Binding Protein (CEBiP) and the Chitin Elicitor Receptor Kinase 1 (CERK1) (Kaku et al., 2006; Shimizu et al., 2010). CEBiP was originally identified as a chitin receptor in rice (OsCEBiP) (Kaku et al., 2006) and is a membrane-anchored extracellular protein that is predicted to be heavily glycosylated (Kaku et al., 2006). The nature and importance of these glycosylations to OsCEBiP function are currently unknown. OsCEBiP recognizes and binds chitin via its extracellular LysM-containing domain but contains no intracellular signaling domain, and is therefore unable to initiate immune signaling alone. OsCERK1 is a transmembrane protein with an extracellular LysM-containing domain and an intracellular kinase domain (Shimizu et al., 2010). OsCERK1 is essential for the generation of a MitogenActivated Protein (MAP) kinase signaling cascade following chitin elicitation (Shimizu et al., 2010). The precise mechanism of chitin binding by OsCEBiP has recently been elucidated and shown to require receptor dimerization (Hayafune et al., 2014).

Wheat contains one CEBiP and two sequence-related CERK1 genes that show high degrees of similarity to their rice orthologs (81 and $86 \%$, respectively) (Lee et al., 2014). Whilst wheat CEBiP and CERK1 are understudied relative to their rice counterparts, their importance during the $Z$. tritici asymptomatic infection phase have been revealed in a recent study (Lee et al., 2014). It was demonstrated that the $Z$. tritici mutant lacking a functional Mg3LysM gene, which normally shows dramatically reduced virulence, was able to regain nearly wild-type virulence on wheat plants in which either CEBiP or CERK1 was silenced through Virus-Induced Gene Silencing (VIGS) (Lee et al., 2014, 2015). This is consistent with the role of the Mg3LysM effector in chitin sequestration (Marshall et al., 2011).

\section{Z. tritici EFFECTORS POTENTIALLY CONTRIBUTING TO THE SECOND, SYMPTOMATIC INFECTION PHASE}

Bioinformatics-based genome-wide approaches have identified several hundred $Z$. tritici genes encoding candidate secreted 


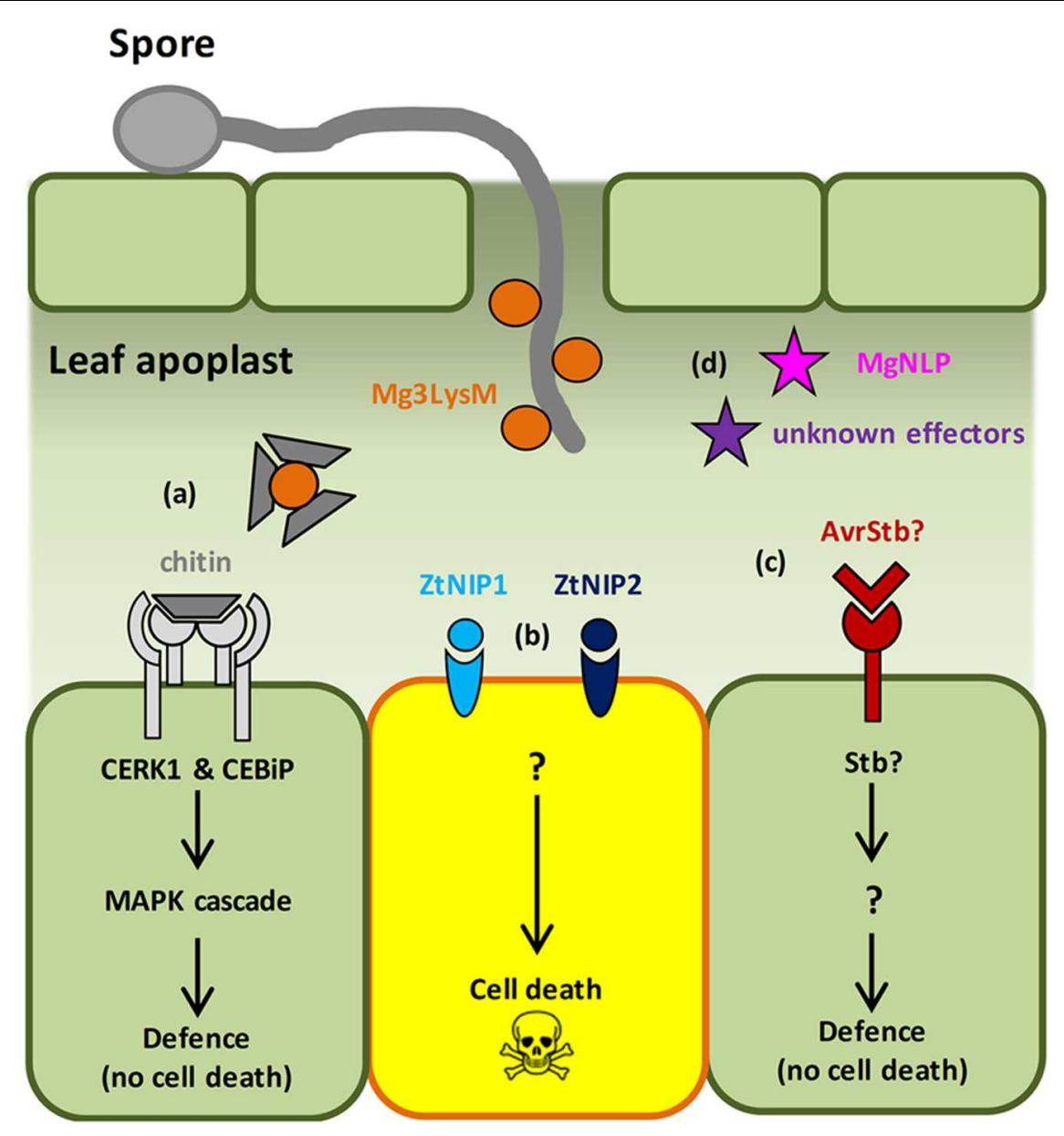

FIGURE 1 | Molecular events in the Zymoseptoria tritici-wheat interaction. (a) Fungal PAMP chitin is recognized by the host receptors Chitin Elicitor Binding Protein (CEBiP) and Chitin Elicitor Receptor Kinase 1 (CERK1), triggering MAP kinase cascades and immune activation. Multi-functional LysM-domain containing effector Mg3LysM scavenges chitin to suppress immunity and protects fungal hyphae from wheat chitinases. (b) 'Necrotrophic' effectors, Necrosis-Inducing Protein 1/2 (ZtNIP1/2), induce host cell death via an unknown mechanism. (c) Stb gene-specified resistance presumably triggered following recognition of cognate fungal effectors (AvrStb) secreted into the apoplast. This results in arrest of pathogen growth via an unknown mechanism that does not involve HR. (d) The Necrosis and Ethylene-Inducing Peptide 1 (NEP1)-like effector protein MgNLP has an unknown function(s) during wheat infection, but triggers cell death in dicotyledonous plants. Unknown effectors $-Z$. tritici effectors predicted from bioinformatic analyses.

proteins that bear hallmarks of effectors identified from other pathosystems, i.e., small, cysteine rich, and expressed in planta (do Amaral et al., 2012; Gohari et al., 2015). Approximately 100 of these candidate effector genes have been shown to peak in expression either during the switch from asymptomatic to symptomatic infection phase, or during the symptomatic phase (Yang et al., 2013b; Rudd et al., 2015). Some of these effectors also become quite abundant during this second phase and can be detected in apoplastic fluids from infected leaf tissues using proteomics analysis (Yang et al., 2013a).

One of the $Z$. tritici effectors transcriptionally upregulated toward the late stage of symptomless colonization by $Z$. tritici is MgNLP, a member of the Necrosis and Ethylene-inducing Peptide 1 (NEP1)-like (NLP) protein family identified and characterized from a number of bacteria, fungi and oomycetes, including many plant pathogens (Gijzen and Nürnberger, 2006). Whilst NLPs often occur in large families, thus making functional redundancy problematic, $Z$. tritici harbors only a single NEP1like protein (Motteram et al., 2009). Similar to other NLPs, MgNLP was found to induce defense-related responses and cell death in leaves of dicotyledonous plants (Motteram et al., 2009). Curiously, MgNLP does not induce defense genes or trigger cell death in wheat, and a targeted $M g N L P$ deletion mutant of $Z$. tritici is not compromised in its ability to infect wheat (Motteram et al., 2009). Therefore, MgNLP may be functionally redundant and its exact function during wheat infection remains elusive (Figure 1).

It has been recently shown that liquid $Z$. tritici culture filtrates contain proteinaceous effectors able to induce cell death when injected into wheat leaves (M'Barek et al., 2015). This study identified two putative 'Necrosis-Inducing Proteins' designated as ZtNIP1 and ZtNIP2 through fast protein liquid chromatography and liquid-chromatography mass spectrometry fractionation of culture filtrates. Syringeinfiltration of heterologously expressed ZtNIP1 and ZtNIP2 into 
wheat leaves induced cell death in a cultivar-dependent fashion (M'Barek et al., 2015). Whilst the specific cell death-inducing activity of these proteins is as yet unknown, these findings point to the existence of interactions between the $Z$. tritici 'necrotrophic' effectors and the corresponding host susceptibility factors (Figure 1), similar to those described for necrotrophic pathogens which infect wheat, Phaeosphaeria nodorum (syn. Stagonospora nodorum) and Pyrenophora tritici-repentis (Tan et al., 2015).

Functional characterisation of other candidate phase-specific $Z$. tritici effectors identified through bioinformatic, proteomic and genome-wide transcriptomic analyses (do Amaral et al., 2012; Gohari et al., 2015; Rudd et al., 2015) is currently underway in several laboratories around the globe including ours, and appears a rich source of future discoveries.

\section{MANAGING Septoria tritici BLOTCH DISEASE IN WHEAT}

The control of STB disease in wheat crops currently relies on the intensive use of fungicides with mixtures of azole and succinate dehydrogenase inhibitor (SDHI) fungicides being most effective (Torriani et al., 2015). Resistance to STB is also an important target in wheat breeding, recently catalyzed by new EU regulations restricting the use of some of the most potent fungicide products as well as increasingly frequent reports of fungicide resistance in field populations of $Z$. tritici $^{1}$. In comprehensive worldwide wheat germplasm screening programs, a number of exotic as well as synthetic wheat genotypes have been identified as good sources of STB resistance. At least 20 distinct genetic loci have been identified (Stb loci) that confer qualitative, often isolate-specific resistance to $Z$. tritici. In addition, a large number of quantitative trait loci (QTL), which make smaller contributions to the $Z$. tritici resistance phenotype, have been mapped genetically. For a comprehensive up-to-date review of both Stb loci and resistance QTLs see Brown et al. (2015).

Although major STB resistance loci (designated as Stb1 through to $S t b 18, S t b S m 3$, and $S t b W W$ ) have been identified in hexaploid wheat, all of these with the exception of Stb16q provide protection against individual or small groups of $Z$. tritici isolates. Stb6 is one of the better-characterized resistance genes. Stb6-based resistance conforms to the gene-for-gene hypothesis, whereby avirulent $Z$. tritici isolates (such as the reference isolate IPO323 with fully sequenced genome; Goodwin et al., 2011) carry the matching AvrStb6 gene (Brading et al., 2002). However, the identity of AvrStb6 is not yet known and so the frequency of this avirulence gene in current $Z$. tritici field populations remains to be determined. Interestingly, Stb6 is found in many commercial wheat cultivars and breeding lines originating from Europe, China, Israel, and the United States (US) that are known sources of $Z$. tritici resistance (Chartrain et al., 2005). This gene appears to contribute some resistance to STB in the

\footnotetext{
${ }^{1}$ http://cereals.ahdb.org.uk/press/2015/december/16/statement-on-sdhi-fungicid es-and-resistance-risk-in-cereals-issued-by-frag-uk.aspx
}

field at least in the United Kingdom (UK) (Arraiano et al., 2009) indirectly suggesting that AvrStb6 may be relatively well represented in regional field populations of $Z$. tritici. Frequencies of avirulence toward other known $S t b$ genes in field populations of the fungus are unknown. However, it is plausible that these may be sufficiently high for some individual Stb genes to provide a certain degree of resistance durability. With this in mind, it is worth noting that US wheat breeders have been actively deploying two major resistance genes, Stb1 and Stb4, since the early 1970s (Goodwin, 2007). Stb1 provided long-lasting resistance to wheat in the central USA (Adhikari et al., 2004), and Stb4 remained effective in California for about 15 years until the effectiveness of this gene decreased (Jackson et al., 2000). Efficacy and durability of the other known $S t b$ genes against natural fungal populations is not known. Importantly, some wheat genotypes used as major sources of resistance to STB in current world breeding programs, for example, TE9111, Kavkaz-K4500 L.6.A.4 and Veranopolis, all contain $\geq 3$ Stb genes (Chartrain et al., 2004; Kollers et al., 2013). This strongly suggests that pyramiding $Z$. tritici isolate-specific resistance genes may be an effective strategy for developing wheat cultivars with high levels of field resistance.

Stb16q is particularly interesting from the disease resistance breeding point of view because this gene appears to confer broad-spectrum seedling stage resistance, as so far no resistancebreaking Z. tritici isolates have been found. Stb16q has been identified in synthetic hexaploid wheat and was shown to originate from one of its parents, the diploid wild wheat species Aegilops tauschii (Tabib Ghaffary et al., 2012). Interestingly, broad-spectrum resistance to $Z$. tritici has also been identified in the diploid wheat Triticum monococcum and was shown to be controlled by a single genetic locus TmStb1 (Jing et al., 2008). Efforts are currently underway to introgress TmStb1 into hexaploid wheat ${ }^{2}$. It would be interesting to evaluate the usefulness of Stb16q and TmStb1 for potentially achieving durable broad-spectrum resistance to $Z$. tritici in bread wheat as well as to explore further the full potential of synthetic hexaploid wheat and wild relatives of wheat as novel sources of resistance to $Z$. tritici.

The functional characterisation of several of the known Stb genes would greatly increase fundamental understanding of the wheat immune system and may facilitate the identification of novel candidate resistance genes using bioinformatic approaches.

\section{FUTURE STRATEGIES FOR STB DISEASE RESISTANCE}

\section{Exploiting PTI to Enhance Resistance to Z. tritici}

The battle that exists in the apoplastic space for chitin binding goes some way to determining the overall outcome of the Z. tritici-wheat interaction. The comparative chitinbinding affinity of the wheat receptors CEBiP and CERK1

\footnotetext{
${ }^{2}$ http://www.wgin.org.uk
} 
relative to chitin-scavenging ability of the fungal effector Mg3LysM is unknown. It is not unreasonable to assume that pathogen effectors such as Mg3LysM, under high evolutionary pressure, might be optimized for chitin binding affinity. One novel strategy, therefore, would be to engineer wheat immune receptors to match or exceed the chitin affinity of the competing fungal effector proteins. Such a tactic might allow 'supercharging' the PTI layer of the wheat immune system. Indeed, such a resource might already exist in nature. However, no study has yet examined the variation in chitin sensitivity across commercial wheat cultivars or their wild relatives.

Furthermore, chitin is likely not the only PAMP present in the apoplastic space during $Z$. tritici infection. $\beta$-glucans are another major components of the fungal cell wall and preparations of these compounds from $Z$. tritici have been shown to elicit defense responses in wheat and to confer some resistance to a normally susceptible cultivar (Shetty et al., 2009). Understanding of how plants perceive $\beta$-glucans is immature in comparison to perception of chitin. Indeed, it is not known if a dedicated membrane-bound immune receptor exists. Nonetheless, enhancement of multiple immune receptors concurrently might offer the same benefits as has been widely discussed with the stacking of dominant isolate- or race-specific resistance $(R)$ genes.

\section{Exploiting Major Resistance Genes for Enhanced Resistance to Z. tritici}

Whilst considerable scientific and breeding efforts have been directed toward the identification of useful sources of $Z$. tritici resistance and development of elite resistant wheat, fully resistant varieties are not yet available to farmers. Taking the UK as an example, the Agriculture and Horticulture Development Board (AHDB) 2016/17 recommended list for winter wheat provides information on the level of disease resistance for current commercially available wheat cultivars ${ }^{3}$. For $Z$. tritici resistance, the vast majority of cultivars (34 of 36) score 6 or less, on a scale of 0-9 (whereby 0 indicates high level of susceptibility and 9 indicates high level of resistance). Only two cultivars (Graham and KWS Siskin) scored as high as 7. This highlights the need for development of novel strategies in breeding for $Z$. tritici resistance.

Single major $S t b$ genes can confer complete resistance to Z. tritici, albeit against a relatively narrow range of isolates harboring the corresponding avirulence (Avr) genes (Figure 1). Deployment of single resistance genes rarely provides durable disease resistance as avirulent isolates of a target pathogen need only to escape this single recognition event in order to regain virulence. Stacking of multiple Stb genes against $Z$. tritici either through marker assited breeding or via cysgenics will likely provide more effective resistance as evidenced by the prevalence of multiple Stb genes in highly resistant germplasm. Biochemical and molecular characterisation of Stb gene products will aid the design of optimal stacking strategies and may also facilitate identification of novel $S t b$ alleles.

\footnotetext{
${ }^{3}$ http://cereals.ahdb.org.uk/varieties/ahdb-recommended-lists.aspx
}

\section{Effector-Assisted Screening for Novel Resistances in Wheat}

For biotrophic pathogens, recognition of an effector by a corresponding major host resistance protein often leads to induction of the hypersensitive response (HR) and localized cell death that correlates with limited pathogen spread beyond the primary inoculated loci. By contrast, recognition of some 'necrotrophic' pathogen effectors triggers induction of host cell death that leads to host susceptibility (Faris et al., 2013). Notable examples are the toxic effectors from the pathogens $P$. nodorum and $P$. tritici-repentis (Ptr) that cause glume blotch and tan spot disease of wheat, respectively (Faris et al., 2013; Vleeshouwers and Oliver, 2014). For Ptr ToxA, effector sensitivity correlates with disease susceptibility across a collection of wheat cultivars (reviewed in Faris et al., 2013). ToxA sensitivity is dependent on the wheat gene $T s n 1$, although there is no evidence of a direct ToxA-Tsn1 interaction (Faris et al., 2010). Nonetheless, screening of Australian wheat germplasm using recombinant ToxA protein has helped direct breeding programs toward the elimination of Ptr susceptibility. Effector-directed breeding reduced the area of ToxA-sensitive wheat grown in Western Australia by nearly $50 \%$ over the first three growing seasons since the program was initiated in 2009 (Vleeshouwers and Oliver, 2014). Similar programs may be initiated to breed out susceptibility to $Z$. tritici. However, knowledge of $Z$. tritici susceptibility genes in wheat and identification of 'necrotrophic' effectors potentially contributing to disease is less advanced than for pathogens such as Ptr. Recent description of $Z$. tritici effectors ZtNIP1 and ZtNIP2 that induce cell death in some but not all wheat cultivars (M'Barek et al., 2015) suggest that sensitivity to 'necrotrophic' effectors may potentially contribute to $Z$. tritici susceptibility. If proven, this may be exploited for breeding resistant (or at least less susceptible) wheat in a similar fashion as discussed above for the wheat-Ptr interaction.

\section{CONCLUSION AND FUTURE PERSPECTIVES}

Molecular characterisation of the major events occurring during $Z$. tritici infection of wheat has enabled more thorough understanding of disease progression. Significant progress has been made, for example, in describing the candidate $Z$. tritici effector repertoire. However, how the $>100$ candidate effectors act in concert to induce disease remains unknown. Likewise, the existence of $S t b$ loci conferring strong isolate-specific resistance has been known for some time, but only one has so far been cloned or functionally characterized (unpublished). Nonetheless, progress that has been made give reason to be optimistic that application of more advanced (and rapid) molecular tools will allow fuller understanding of STB disease development, taking it on par with model pathogens.

Where perhaps a greater problem lies, however, is the translation of fundamental findings into real-world impact. But even here, trails have been blazed using other pathosystems that may point the way for $Z$. tritici research. The implementation 
of an effector-directed breeding program against Ptr (discussed above) is a prime example of how fundamental research can lead to low-cost, time-saving tools for the benefit of agriculture.

It is speculated that introduction of $Z$. tritici susceptibility into breeding programs came as a result of breeding for higher yields combined with tight association of gene loci conditioning susceptibility and those for yield potential (Torriani et al., 2015). In the event that culprit susceptibility gene loci are identified and characterized biochemically in wheat, genome editing technologies such as CRISPR-Cas9 (Wang et al., 2014) might be used in their modification or inactivation without the necessary yield loss penalties demanded by agriculture.

\section{AUTHOR CONTRIBUTIONS}

GK and KK jointly conceived this mini review. GK produced the first draft and GK and KK then critically revised the manuscript. Both, GK and KK approve the final version of the manuscript.

\section{REFERENCES}

Adhikari, T. B., Yang, X., Cavaletto, J. R., Hu, X., Buechley, G., Ohm, H. W., et al. (2004). Molecular mapping of Stb1, a potentially durable gene for resistance to Septoria tritici blotch in wheat. Theor. Appl. Genet. 109, 944-953. doi: 10.1007/s00122-004-1709-6

Arraiano, L. S., Balaam, N., Fenwick, P. M., Chapman, C., Feuerhelm, D., Howell, P., et al. (2009). Contributions of disease resistance and escape to the control of Septoria tritici blotch of wheat. Plant Pathol. 58, 910-922. doi: 10.1111/j.1365-3059.2009.02118.x

Bolton, M. D., van Esse, H. P., Vossen, J. H., de Jonge, R., Stergiopoulos, I., Stulemeijer, I. J., et al. (2008). The novel Cladosporium fulvum lysin motif effector Ecp6 is a virulence factor with orthologues in other fungal species. Mol. Microbiol. 69, 119-136. doi: 10.1111/j.1365-2958.2008.06270.x

Brading, P. A., Verstappen, E. C. P., Kema, G. H. J., and Brown, J. K. M. (2002). A gene-for-gene relationship between wheat and Mycosphaerella graminicola, the Septoria tritici blotch pathogen. Phytopathology 92, 439-445. doi: 10.1094/PHYTO.2002.92.4.439

Brown, J. K., Chartrain, L., Lasserre-Zuber, P., and Saintenac, C. (2015). Genetics of resistance to Zymoseptoria tritici and applications to wheat breeding. Fungal Genet. Biol. 79, 33-41. doi: 10.1016/j.fgb.2015.04.017

Chartrain, L., Brading, P. A., and Brown, J. K. M. (2005). Presence of the Stb6 gene for resistance to Septoria tritici blotch (Mycosphaerella graminicola) in cultivars used in wheat-breeding programmes worldwide. Plant Pathol. 54, 134-143. doi: 10.1111/j.1365-3059.2005.01164.x

Chartrain, L., Brading, P. A., Makepeace, J. C., and Brown, J. K. M. (2004). Sources of resistance to Septoria tritici blotch and implications for wheat breeding. Plant Pathol. 53, 454-460. doi: 10.1111/j.1365-3059.2004.01052.x

de Jonge, R., van Esse, H. P., Kombrink, A., Shinya, T., Desaki, Y., Bours, R., et al. (2010). Conserved fungal LysM effector Ecp6 prevents chitintriggered immunity in plants. Science 329, 953-955. doi: 10.1126/science.11 90859

do Amaral, A. M., Antoniw, J., Rudd, J. J., and Hammond-Kosack, K. E. (2012). Defining the predicted protein secretome of the fungal wheat leaf pathogen Mycosphaerella graminicola. PLoS ONE 7:e49904. doi: 10.1371/journal.pone.0049904

Faris, J. D., Liu, Z., and Xu, S. S. (2013). Genetics of tan spot resistance in wheat. Theor. Appl. Genet. 126, 2197-2217. doi: 10.1007/s00122-013-2157-y

Faris, J. D., Zhang, Z., Lu, H., Lu, S., Reddy, L., Cloutier, S., et al. (2010). A unique wheat disease resistance-like gene governs effector-triggered susceptibility to necrotrophic pathogens. Proc. Natl. Acad. Sci. U.S.A. 107, 13544-13549. doi: 10.1073/pnas. 1004090107
Both authors agree to be accountable for all aspects of the manuscript in ensuring that questions related to the accuracy or integrity of any part of the work are appropriately investigated and resolved.

\section{FUNDING}

Research was funded by the Institute Strategic Programme Grant "20:20 Wheat ${ }^{\circledR}$ " (grant no. BB/J/00426X/1) from the Biotechnology and Biological Sciences Research Council of the UK (BBSRC) and by the Rothamsted Institute Fellowship Programme.

\section{ACKNOWLEDGMENTS}

We would like to thank Kim E. Hammond-Kosack for critical reading of this manuscript and helpful comments.

Gijzen, M., and Nürnberger, T. (2006). Nep1-like proteins from plant pathogens: recruitment and diversification of the NPP1 domain across taxa. Phytochemistry 67, 1800-1807. doi: 10.1016/j.phytochem.2005.12.008

Gohari, A. M., Ware, S. B., Wittenberg, A. H., Mehrabi, R., M'Barek, S. B., Verstappen, E. C. P., et al. (2015). Effector discovery in the fungal wheat pathogen Zymoseptoria tritici. Mol. Plant Pathol. 16, 931-945. doi: $10.1111 / \mathrm{mpp} .12251$

Goodwin, S. B. (2007). Back to basics and beyond: increasing the level of resistance to Septoria tritici blotch in wheat. Australas. Plant Pathol. 36, 532-538. doi: 10.1071/AP07068

Goodwin, S. B., M'barek, S. B., Dhillon, B., Wittenberg, A. H. J., Crane, C. F., Hane, J. K., et al. (2011). Finished genome of the fungal wheat pathogen Mycosphaerella graminicola reveals dispensome structure, chromosome plasticity, and stealth pathogenesis. PLoS Genet. 7:e1002070. doi: 10.1371/journal.pgen.1002070

Hayafune, M., Berisio, R., Marchetti, R., Silipo, A., Kayama, M., Desaki, Y., et al. (2014). Chitin-induced activation of immune signaling by the rice receptor CEBiP relies on a unique sandwich-type dimerization. Proc. Natl. Acad. Sci. U.S.A. 111, E404-E413. doi: 10.1073/pnas.1312099111

Jackson, L. F., Dubcovsky, J., Gallagher, L. W., Wennig, R. L., Heaton, J., Vogt, R. L., et al. (2000). 2000 regional barley and common and durum wheat performance tests in California. UC Davis Agron. Prog. Rep. 272, 1-56.

Jing, H. C., Lovell, D., Gutteridge, R., Jenk, D., Kornyukhin, D., Mitrofanova, O. P., et al. (2008). Phenotypic and genetic analysis of the Triticum monococcumMycosphaerella graminicola interaction. New Phytol. 179, 1121-1132. doi: 10.1111/j.1469-8137.2008.02526.x

Jones, J. D. G., and Dangl, J. L. (2006). The plant immune system. Nature 444, 323-329. doi: 10.1038/nature05286

Kaku, H., Nishizawa, Y., Ishii-Minami, N., Akimoto-Tomiyama, C., Dohmae, N., Takio, K., et al. (2006). Plant cells recognize chitin fragments for defense signaling through a plasma membrane receptor. Proc. Natl. Acad. Sci. U.S.A. 103, 11086-11091. doi: 10.1073/pnas.0508882103

Kema, G. H. J., Yu, D. Z., Rijkenberg, H. J., Shaw, M. W., and Baayen, R. P. (1996). Histology of the pathogenesis of Mycosphaerella graminicola in wheat. Phytopathology 86, 777-786. doi: 10.1094/Phyto-86-777

Keon, J., Antoniw, J., Carzaniga, R., Deller, S., Ward, J. L., Baker, J. M., et al. (2007). Transcriptional adaptation of Mycosphaerella graminicola to programmed cell death (PCD) of its susceptible wheat host. Mol. Plant Microbe Interact. 20, 178-193. doi: 10.1094/MPMI-20-2-0178

Klarzynski, O., Plesse, B., Joubert, J. M., Yvin, J. C., Kopp, M., Kloareg, B., et al. (2000). Linear beta-1,3 glucans are elicitors of defense responses in tobacco. Plant Physiol. 124, 1027-1038. doi: 10.1104/pp.124.3.1027 
Kollers, S., Rodemann, B., Ling, J., Korzun, V., Ebmeyer, E., Argillier, O., et al. (2013). Genetic architecture of resistance to Septoria tritici blotch (Mycosphaerella graminicola) in European winter wheat. Mol. Breed. 32, 411423. doi: 10.1007/s11032-013-9880-6

Lee, W. S., Rudd, J. J., Hammond-Kosack, K. E., and Kanyuka, K. (2014). Mycosphaerella graminicola LysM effector-mediated stealth pathogenesis subverts recognition through both CERK1 and CEBiP homologues in wheat. Mol. Plant Microbe Interact. 27, 236-243. doi: 10.1094/MPMI-07-130201-R

Lee, W. S., Rudd, J. J., and Kanyuka, K. (2015). Virus induced gene silencing (VIGS) for functional analysis of wheat genes involved in Zymoseptoria tritici susceptibility and resistance. Fungal Genet. Biol. 79, 84-88. doi: 10.1016/j.fgb.2015.04.006

Lochman, J., and Mikes, V. (2006). Ergosterol treatment leads to the expression of a specific set of defence-related genes in tobacco. Plant Mol. Biol. 62, 43-51. doi: 10.1007/s11103-006-9002-5

Marshall, R., Kombrink, A., Motteram, J., Loza-Reyes, E., Lucas, J., HammondKosack, K. E., et al. (2011). Analysis of two in planta expressed LysM effector homologs from the fungus Mycosphaerella graminicola reveals novel functional properties and varying contributions to virulence on wheat. Plant Physiol. 156, 756-769. doi: 10.1104/pp.111.176347

M'Barek, B. S., Cordewener, J., Ghaffary, S. M. T., Lee, T. V. D., Liu, Z., Gohari, A. M., et al. (2015). FPLC and liquid-chromatography mass spectrometry identify candidate necrosis-inducing proteins from culture filtrates of the fungal wheat pathogen Zymoseptoria tritici. Fungal Genet. Biol. 79, 54-62. doi: 10.1016/j.fgb.2015.03.015

McGreal, E. P., Rosas, M., Brown, G. D., Zamze, S., Wong, S. Y., Gordon, S., et al. (2006). The carbohydrate-recognition domain of Dectin-2 is a C-type lectin with specificity for high mannose. Glycobiology 16, 422-430. doi: $10.1093 /$ glycob/cwj077

Motteram, J., Küfner, I., Deller, S., Brunner, F., Hammond-Kosack, K. E., Nürnberger, T., et al. (2009). Molecular characterization and functional analysis of MgNLP, the sole NPP1 domain-containing protein, from the fungal wheat leaf pathogen Mycosphaerella graminicola. Mol. Plant Microbe Interact. 22, 790-799. doi: 10.1094/MPMI-22-7-0790

Rudd, J. J., Kanyuka, K., Hassani-Pak, K., Derbyshire, M., Andongabo, A., Devonshire, J., et al. (2015). Transcriptome and metabolite profiling the infection cycle of Zymoseptoria tritici on wheat (Triticum aestivum) reveals a biphasic interaction with plant immunity involving differential pathogen chromosomal contributions, and a variation on the hemi-biotrophic lifestyle definition. Plant Physiol. 167, 1158-1185.

Sánchez-Vallet, A., Mesters, J. R., and Thomma, B. P. (2014). The battle for chitin recognition in plant-microbe interactions. FEMS Microbiol. Rev. 39, 171-183. doi: 10.1093/femsre/fuu003
Shetty, N. P., Jensen, J. D., Knudsen, A., Finnie, C., Geshi, N., Blennow, A., et al. (2009). Effects of beta-1,3-glucan from Septoria tritici on structural defence responses in wheat. J. Exp. Bot. 60, 4287-4300. doi: 10.1093/jxb/erp269

Shimizu, T., Nakano, T., Takamizawa, D., Desaki, Y., Ishii-Minami, N., Nishizawa, Y., et al. (2010). Two LysM receptor molecules, CEBiP and OsCERK1, cooperatively regulate chitin elicitor signaling in rice. Plant J. 64, 204-214. doi: 10.1111/j.1365-313X.2010.04324.x

Tabib Ghaffary, S. M., Faris, J. D., Friesen, T. L., Visser, R. G., van der Lee, T. A., Robert, O., et al. (2012). New broad-spectrum resistance to Septoria tritici blotch derived from synthetic hexaploid wheat. Theor. Appl. Genet. 124, 125-142. doi: 10.1007/s00122-011-1692-7

Tan, K. C., Phan, H. T., Rybak, K., John, E., Chooi, Y. H., Solomon, P. S., et al. (2015). Functional redundancy of necrotrophic effectors - consequences for exploitation for breeding. Front. Plant Sci. 6:501. doi: 10.3389/fpls.2015.00501

Torriani, S. F. F., Melichar, J. P. E., Mills, C., Pain, N., Sierotzki, H., and Courbot, M. (2015). Zymoseptoria tritici: a major threat to wheat production, integrated approaches to control. Fungal Genet. Biol. 79, 8-12. doi: 10.1016/j.fgb.2015.04.010

Vleeshouwers, V. G., and Oliver, R. P. (2014). Effectors as tools in disease resistance breeding against biotrophic, hemibiotrophic, and necrotrophic plant pathogens. Mol. Plant Microbe Interact. 27, 196-206. doi: 10.1094/MPMI-1013-0313-IA

Wang, Y., Cheng, X., Shan, Q., Zhang, Y., Liu, J., Gao, C., et al. (2014). Simultaneous editing of three homoeoalleles in hexaploid bread wheat confers heritable resistance to powdery mildew. Nat. Biotechnol. 32, 947-951. doi: $10.1038 /$ nbt.2969

Yang, F., Li, W., Derbyshire, M., Larsen, M. R., Rudd, J. J., and Palmisano, G. (2013a). Unraveling incompatibility between wheat and the fungal pathogen Zymoseptoria tritici through apoplastic proteomics. BMC Genomics 16:362. doi: 10.1186/s12864-015-1549-6

Yang, F., Li, W., and Jørgensen, H. J. L. (2013b). Transcriptional reprogramming of wheat and the hemibiotrophic pathogen Septoria tritici during two phases of the compatible interaction. PLoS ONE 8:e81606. doi: 10.1371/journal.pone.0081606

Conflict of Interest Statement: The authors declare that the research was conducted in the absence of any commercial or financial relationships that could be construed as a potential conflict of interest.

Copyright $\odot 2016$ Kettles and Kanyuka. This is an open-access article distributed under the terms of the Creative Commons Attribution License (CC BY). The use, distribution or reproduction in other forums is permitted, provided the original author(s) or licensor are credited and that the original publication in this journal is cited, in accordance with accepted academic practice. No use, distribution or reproduction is permitted which does not comply with these terms. 